\title{
Solving Differential Equations by New Wavelet Type Transform Method Based on the Wavelets and Symmetry Groups
}

\author{
Yazdani $\mathrm{HR}^{1 *}$ and Nadjafikhah $\mathrm{M}^{2}$
}

${ }^{1}$ Department of Mathematics, Payame Noor University (PNU), P.O. Box 19395-3697, Tehran, Iran

${ }^{2}$ School of Mathematics, Iran University of Science and Technology, Narmak, Tehran, Iran

\begin{abstract}
The wavelets are important functions in the harmonic analysis. Up to our knowledge, apply wavelets to solve differential equations are limited to ODEs or PDEs with approximate and numerical solutions. In this paper, the novel methods based on the wavelets with two independent variables according to differential invariants are proposed. In fact, the transform groups are constructed that can be acted on the existing solutions and produced the new solution. These groups are based on the symmetry groups (obtained by the Lie symmetry method and other equivalence methods) and mother wavelets. The new method based on the wavelets are presented, new mother wavelets are produced, the corresponding wavelet type transform groups are provided and applied for solving the differential equations. Our method can be used for ODEs and PDEs at every order and give us the analytic solutions according to the existing solutions (from every method without any exception).
\end{abstract}

Keywords: Wavelet; Mother wavelet; Wavelet type transform; Invariant solution; Degree reduction

\section{Introduction}

The wavelets are very useful tools for analyzing problems. They are important functions in the functional and harmonic analysis. The first wavelet was introduced by Alphered Haar (the Hungarian mathematician) [1]. Nowadays, the wavelets have numerous applications in some fields of science and technology; such as seismology, image processing, signal processing, coding theory, biosciences, financial mathematics, fractals and so on [2]. The application of wavelets for solving differential equations limited to ODEs or PDEs with the numerical solutions in the special conditions. The famous wavelets such as Haar, Daubechie, Coiflet, Symlet, CDF, Mexican hat and Gaussian are extendible to two or more variables with tensor product. The wavelets with two or more variables (that in connection with PDEs are useful) is very important. In this paper, we make some new wavelets with two variables in different method; these wavelets depend on the differential invariants of differential equations. Therefore, we can use their transform groups for solving differential equations. This method called the Wavelet Type Transform Method (WTTM). We will show the performance of WTTM with examples.

We propose the method based on the wavelets with two independent variables according to the symmetry groups. This method based on the group action of wavelet transforms correspond to the suitable mother wavelets on the differential equations. In fact, by wavelet transforms, we act on the solution spaces obtained by other methods (like the Lie symmetry method) and construct the new solutions according to the existing solutions. For this reason, this method is called the wavelet type transform method (WTTM). Recently, the similar method based on the Fourier transforms has been proposed by Craddock [3]. Here, first briefly explain the Lie symmetry method that will be applied for obtaining the differential invariants.

The Lie symmetry groups theory of differential equations was developed by Sophus Lie [2]. Such Lie groups are invertible point transforms of both the dependent and independent variables of the differential equations. The symmetry group method provide the collection of tools for analyzing differential equations and is of great importance to understand and to construct solutions of differential equations. Several applications of Lie groups in the theory of differential equations were discussed in the literature, the most important ones are: the reduction of order of ordinary differential equations (ODEs), the construction of invariant solutions, mapping the solutions to other solutions and the detection of linear transforms $[4,5]$.

The remainder of this paper is organized as follows. In section 2, we recall some needed results to construct differential invariants, the mother wavelets and the wavelet type transforms. In section 3, the wavelet type transform method (WTTM) is proposed. In sections 4, the proposed method will be demonstrated by examples. Finally, the conclusions and future works are presented.

\section{Preliminaries}

In this section, we remember some definitions, conceptions and theorems from related areas.

\section{The Lie symmetry method}

In this section, we recall the general procedure for determining symmetries for any system of partial differential equations $[2,4,6,7]$.

To begin, let us consider the general case of a nonlinear system of partial differential equations of order $n$th in $p$ independent and $q$ dependent variables is given as a system of equations:

$$
\Delta_{v}\left(x, u^{(n)}\right)=0, v=1, \cdots, l,
$$

Involving $x=\left(x^{1}, \cdots, x^{p}\right), u=\left(u^{1}, \cdots, u^{q}\right)$ and the derivatives of $u$ with respect to $x$ up to $\mathrm{n}$, where $u^{(n)}$ represents all the derivatives of $u$ of all orders from 0 to $n$. We consider a one-parameter Lie group of infinitesimal transforms acting on the independent and dependent variables of the system (1) as below:

*Corresponding author: Yazdani HR, Department of Mathematics, Payame Noor University (PNU), P.O. Box 19395-3697, Tehran, Iran, Tel: +98 212332 0000; E-mail: yazdani@phd.pnu.ac.ir

Received June 23, 2017; Accepted July 21, 2017; Published July 29, 2017

Citation: Yazdani HR, Nadjafikhah M (2017) Solving Differential Equations by New Wavelet Type Transform Method Based on the Wavelets and Symmetry Groups. J Generalized Lie Theory Appl 11: 272. doi: 10.4172/1736-4337.1000272

Copyright: (c) 2017 Yazdani HR, et al. This is an open-access article distributed under the terms of the Creative Commons Attribution License, which permits unrestricted use, distribution, and reproduction in any medium, provided the original author and source are credited. 


$$
\left(\tilde{x}^{i}, \tilde{u}^{j}\right)=\left(x^{i}, u^{i}\right)+s\left(\xi^{i}, \eta^{j}\right)+O\left(s^{2}\right), \quad(i=1 \cdots, p, \quad j=1 \cdots, q,)
$$

Where $s$ is the parameter of the transform and $\xi^{i}, \eta^{j}$ are the infinitesimals of the transforms for the independent and dependent variables, respectively. The infinitesimal generator $v$ associated with the group of transforms can be written as $v=\sum_{i=1}^{p} \xi^{i} \partial_{x^{i}}+\sum_{j=1}^{q} \eta^{j} \partial_{u^{j}}$, A symmetry of a differential equation is a transform which maps solutions of the equation to other solutions. The invariance of the system (1) under the infinitesimal transforms leads to the invariance conditions (Theorem 2.36 of ref. [4]):

$$
\operatorname{Pr}^{(n)} v\left[\Delta_{i}\left(x, u^{(n)}\right)\right]=0, \quad \Delta_{i}\left(x, u^{(n)}\right)=0, \quad v=1, \cdots, l,
$$

where $\operatorname{Pr}^{(n)}$ is called the $n^{\text {th }}$-order prolongation of the infinitesimal generator and given by $\operatorname{Pr}^{(n)} v=v+\sum_{\alpha=1}^{q}, \sum_{J} \phi_{J}^{\alpha}\left(x, u^{(n)}\right) \partial_{u_{J}^{\alpha}} \mathrm{z}$, where $J=\left(j_{1}, \cdots, j_{k}\right), 1 \leq j_{k} \leq p, 1 \leq k \leq n$ and the sum is over all $\rho$ 's of order $0<\# J \leq n$. If $\# J=k$, the coefficient $\phi_{J}^{\alpha}$ of $\partial_{u_{J}^{\alpha}}$ will only depend on $k$-th and lower order derivatives of $u$, and $\phi_{\alpha}^{J}\left(x, u^{(n)}\right)=D_{J}\left(\phi_{\alpha}-\sum_{i=1}^{p} \xi^{i} u_{i}^{\alpha}\right)+\sum_{i=1}^{p} \xi^{i} u_{J, i}^{\alpha}$, where $u_{i}^{\alpha}:=\partial u^{\alpha} / \partial x^{i}$ and $u_{J, i}^{\dot{a}}:=\partial u_{J}^{\dot{a}} / \partial x^{i}$.

One of the most important properties of these infinitesimal symmetries is that they form a Lie algebra under the usual Lie bracket. The first application of symmetry group methods is to construct the new solutions from known existing solutions. The second is when a nonlinear system of differential equations admits infinite symmetries, so it is possible to transform it to a linear system. Infact, for every vector field, we establish the following characteristics system.

$$
\frac{d x}{\xi}=\frac{d t}{\tau}=\frac{d u}{\phi}
$$

By solving the system, we obtain differential invariants corresponding to the vector fields. The PDE is expressed in the coordinates $(\mathrm{x}, \mathrm{t}, \mathrm{u})$, so for reducing this equation, we should search for its form in the specific coordinates. Those coordinates will be constructed by searching for independent invariants $(\mathrm{y}, \mathrm{v})$ corresponding to the infinitesimal generators. Thus by using the chain rule, the expression of equation in the new coordinate, allows us to reduce the order of the differential equation [6].

\section{The wavelet theory}

The wavelets are important functions in the mathematics and other scientific fields. In this section, we introduce wavelets as functions belong to $L^{2}\left(R^{2}\right)$ (The space of squared integrable functions with integral norm).

Definition 1: The function $\psi$ is a wavelet, if it satisfies in the following admissible condition:

$$
C_{\psi}=\int_{R^{2}} \frac{|F(\psi)(\omega)|^{2} d \omega}{|\omega|}<\infty .
$$

Where $F(\psi)(\omega)$ is the Fourier transform of wavelet $\psi$ and defined as follows:

$$
F(\psi)(\omega)=\frac{1}{\sqrt{2 \pi}} \int_{R^{2}} \exp (-i x \cdot \omega) \psi(x) d \omega z,
$$

$C_{\psi}$ is called the wavelet coefficient of $\psi$. Here, $\omega=\left(\omega_{1}, \omega_{2}\right)$ and $x=\left(x_{1}, x_{2}\right)$ belong to $R_{2}[7]$.
Definition 2: The wavelet $\psi$ is called mother wavelet, if it satisfies in the following properties,

$$
\begin{aligned}
& \int_{R^{2}} \psi(x) d x=0 \\
& \int_{R^{2}}|\psi(x)|^{2} d x<\infty \\
& \lim _{|\omega| \rightarrow \infty} F(\psi(\omega))=0
\end{aligned}
$$

Note that, the first property equivalents to $C_{\psi}>0$ (the admissible condition) for mother wavelet $\psi[1]$.

Indeed, the mother wavelets have the admissible condition, n-zero moments and exponential decay properties. The mother wavelet have two parameters: the translation parameter $b=\left(b_{1}, b_{2}\right)$ and scaling parameter $a>0$. The mother wavelet corresponding to $(a, b)$ is:

$$
\psi_{a, b}(x)=\psi\left(\frac{x-b}{a}\right)=\psi\left(\frac{x_{1}-b_{1}}{a}, \frac{x_{2}-b_{2}}{a}\right)
$$

If the function $\psi$ don't satisfy in the some properties of mother wavelets, or approximately satisfies, $\psi$ is called quasi-wavelet. The quasi-wavelets have numerous applications in the applied mathematics and other scientific fields for solving PDEs [8].

Definition 3: The wavelet transform corresponding to the mother wavelet $\psi$ for the function $f \in L^{2}\left(R^{2}\right)$ with parameters $(a, b)$ defined as follows:

$$
W_{\psi}(f)(a, b)=\frac{1}{\sqrt{|a| \cdot C_{\psi}}} \int_{R^{2}} \psi_{a, b}(x) \cdot f(x) d x .
$$

Thus, the wavelet transform depends on the wavelet $\psi$, the function $\mathrm{f}$, and the parameters $(\mathrm{a}, \mathrm{b})$.

Theorem 1: The wavelet transform as integral operator is from $L^{2}$ $\left(R^{2}\right)$ to $L^{2}\left(R^{3}\right)$ that satisfies in the following properties:

- Linearity: $W_{\psi}[\alpha f(x)+\beta g(x)]=\alpha W_{\psi}[f(x)]+\beta W_{\psi}[g(x)]$,

- Translation: $W_{\psi}[f(x-k)]=W[f(x)](a, b-k), \quad \forall k \in R^{2}$,

- Scaling: $W_{\psi}\left[\frac{1}{\sqrt{s}} f\left(\frac{x}{s}\right)\right]=W[f(x)]\left(\frac{a}{s}, \frac{b}{s}\right)$,

- Wavelet shifting: $W_{\psi(x-k)}[f(x)]=W[f(x)](a, b+a k)$,

- Linear combination: $W_{\hat{a} \psi_{1}+\hat{a} \psi_{2}}[f(x)]=\alpha W_{\psi_{1}}[f(x)]+\beta W_{\psi_{2}}[f(x)]$,

- Wavelet scaling: $W_{\frac{\psi(x / s)}{\sqrt{(\mid s)}}}[f(x)]=W[f(x)](a s, b)$.

Proof [1]: Indeed, the wavelet transforms are isometries [7,9], this property and the admissible condition imply that the wavelet transform is invertible. The inversion formula for the wavelet transform $W_{\psi}(f)$ is:

$$
f(x)=f\left(x_{1}, x_{2}\right)=\frac{1}{C_{\psi}} \int_{R^{+} \times R^{2}}, W_{\psi} f(a, b) \psi_{a, b}(x) \frac{d a d b_{1} d b_{2}}{a^{3}}
$$

In fact, for obtaining the function $\mathrm{f}(\mathrm{x})$ corresponds to the wavelet transform $W_{\psi}(f)$, the inversion formula (also called the synthesis formula) could be employed.

\section{The Wavelet Type Transform Method}

The wavelet type transform method (WTTM) has 3 following steps: 
Citation: Yazdani HR, Nadjafikhah M (2017) Solving Differential Equations by New Wavelet Type Transform Method Based on the Wavelets and Symmetry Groups. J Generalized Lie Theory Appl 11: 272. doi: 10.4172/1736-4337.1000272

Page 3 of 4

- Apply equivalence algorithms (for example, the Lie symmetry method) on DE, and obtain the general solutions as follows:

$$
\tilde{u}(x, t ; \varepsilon):=\rho\left(\exp \left(\varepsilon v_{k}\right)\right) u_{0},
$$

- Propose the suitable mother wavelet (as a test function) based on the symmetry groups and differential invariants. According to mother wavelet $\psi$, similar to the wavelet transforms definition, we have:

$$
U(x, t)=\int, \psi(x, t ; \varepsilon) \cdot \rho\left(\exp \left(\varepsilon v_{k}\right)\right) u_{0}(x, t ; \varepsilon) d \varepsilon,
$$

This is a wavelet type transform that its kernel is an invariant solution of differential equation.

- For every generator vector field $v_{k}$ from the symmetry groups, by considering the suitable values $(0,1)$ for the parameters $\varepsilon_{i}$ (this parameter for symmetry group $G_{i}$ equals 1 and for every symmetry group $G_{j}$ such that $\mathrm{j} \neq \mathrm{i}$ equals 0 ), and the correspondent version of mother wavelet $\psi$ based on the differential invariants of the symmetry groups $G_{i}$ (that's $\left.\psi\left(x, t ; \varepsilon_{i}\right)\right)$, construct $U(x, t)$.

Indeed, $U(x, t)$ is a wavelet transform and on the other hand (for every invariant solution $\tilde{u}(x, t ; \varepsilon))$, it is a wavelet invariant solution.

This method provides a link between harmonic and wavelet analysis and Lie symmetries. In fact, the new solutions can be constructed by integral kernel operators, where the kernels are invariant solutions (obtained by the symmetries of the equation) and test functions are suitable mother wavelets (constructed based on the differential invariants). Therefore, this method converges to the solution $\mathrm{d}$ of differential equations.

\section{Examples}

Here, we demonstrate WTTM by examples. We implement WTTM on the heat, KdV and GFKPP equations and obtain solutions. finally, WTTM results will be proposed. First, we should apply the Lie symmetry method on these equations, for more details and calculations [4]. The following table proposes the mother wavelets according to the differential invariants of symmetry groups: (Table 1).

By the little computation, it can be seen that offered functions have properties (2)-(3) of the mother wavelets. Figure 1 shows the graphs of mother wavelets. The some properties are clear from this figure (some computations and plots done by the maple 2016).

We know that the general solution (from the Lie symmetry method) of the heat equation $u_{t}=u_{x x}$ as follows:

$$
\tilde{u}(x, t ; \varepsilon)=\frac{\exp \left(\varepsilon_{3}-\frac{\varepsilon_{5} x+\varepsilon_{6} x^{2}-\varepsilon_{5}^{2} t}{1+4 \varepsilon_{6} t}\right)}{\sqrt{1+4 \varepsilon_{6} t}} u\left(\frac{\exp \left(-\varepsilon_{4}\right)\left(x-2 \varepsilon_{5} t\right)}{1+4 \varepsilon_{6} t}, \frac{\exp \left(-2 \varepsilon_{4}\right)}{1+4 \varepsilon_{6} t}-\varepsilon_{2}\right),
$$

Thus for the translation symmetry group in the line of $x$, all $\varepsilon s$ (except $i=1$ ) are zero. So,

$$
\tilde{u}(x, t ; \varepsilon)=u\left(x-\varepsilon_{1}, t\right) \text {. }
$$

But for the vector field $v_{1}=\partial_{x}+2 \partial_{t}$, with the differential invariant $(x-2 t)$ (the translation parameter $c=2$ ), we have:

$$
\tilde{u}(x, t ; \varepsilon)=u\left(x-2 \varepsilon_{1}, t-\varepsilon_{2}\right) .
$$

for the corresponding mother wavelet $\psi_{1}(x, t)=\frac{4}{5} \exp \left(-\frac{x^{2}+t^{2}}{2}\right) \sin \left(\frac{\pi(x-2 t)}{2}\right)$, we get,

$$
\psi_{1}(x, t ; \varepsilon)=\frac{4}{5} \exp \left(-\frac{\left(x-2 \varepsilon_{1}\right)^{2}+\left(t-\varepsilon_{2}\right)^{2}}{2}\right) \sin \left(\frac{\pi\left(x-2 \varepsilon_{1}-2 t+2 \varepsilon_{2}\right)}{2}\right)
$$

Therefore:

$$
\iint_{\mathbb{R}^{+} \mathbb{R}^{+}} \frac{4}{5} \exp \left(-\frac{\left(x-2 \varepsilon_{1}\right)^{2}+\left(t-\varepsilon_{2}\right)^{2}}{2}\right) \sin \left(\frac{\pi A}{2}\right) \tilde{u}(x, t ; \varepsilon) d \varepsilon_{1} d \varepsilon_{2} .
$$

Where $A=\left(x-2 t+2\left(\varepsilon_{2}-\varepsilon_{1}\right)\right)$. Indeed $U$ is the wavelet transform $W_{\theta_{1}}(x, t)$ on the related symmetry group $v_{1}$ that its invariant solution as below:

$$
\tilde{u}(x, t ; \varepsilon)=k \exp (-2 A)+l,
$$

By replacing this solution in the integral (5) and integration respect to $\varepsilon_{1}, \varepsilon_{2}$, we get to the wavelet invariant solution of heat equation based on $\psi_{1}(x, t)$. The calculation for mother wavelet $\psi_{2}$ is similar.

Now, let us consider mother wavelet $\psi_{4}$ related to the Galilean boost vector field $v_{4}=2 t \partial_{x} x u \partial_{u}$ with differential invariant solution

\begin{tabular}{|c|c|c|}
\hline Symmetry group & Differential invariants & Mother wavelets \\
\hline Translation & $x c t, u$ & $\left.(4 / 5) \exp \left(\left(x^{2}+t^{2}\right) / 2\right) \sin (\pi(x-c t)) / 2\right)$ \\
\hline Scaling & & $\left.(4 / 5) \exp \left(-\left(x^{2}+t^{2}\right) / 2\right) \cos (\pi(x c t)) / 2\right)$ \\
\hline Galilean Boost & $(x / t),(x / \sqrt{t}),\left(u / t^{2}\right)$ & $\exp \left(-\left(x^{2}+15 t^{2}\right) / 20\right) \cos (x / t) \sin (x / t)$ \\
\hline & $t, u \exp \left(x^{2} / 4 t\right)$ & $\exp \left(-\left(x^{2}+a t^{2}\right) / b\right) \cos (x / t) \sin (x / t)$ \\
\hline
\end{tabular}

Table 1: Symmetry groups, Differential invariants and Mother wavelets.

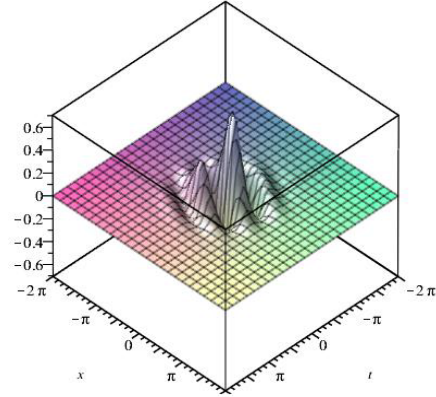

(a) The graph of $\psi_{1}$

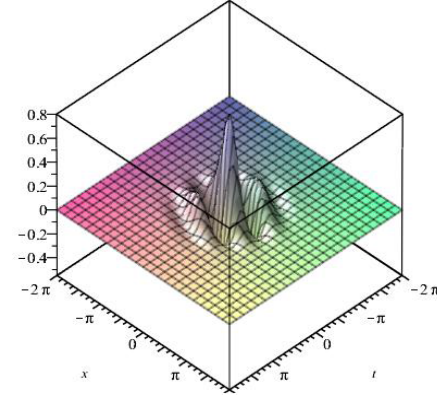

(b) The graph of $\psi_{2}$

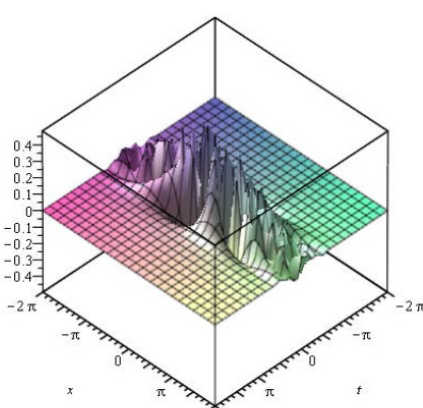

(c) The graph of $\psi_{3}$

Figure 1: The graphs of mother wavelets. 
Citation: Yazdani HR, Nadjafikhah M (2017) Solving Differential Equations by New Wavelet Type Transform Method Based on the Wavelets and Symmetry Groups. J Generalized Lie Theory Appl 11: 272. doi: 10.4172/1736-4337.1000272

$u(x, t)=\frac{k}{\sqrt{t}} \exp \left(-\frac{x^{2}}{4 t}\right)$. The correspondent wavelet type transform as follows:

$$
\int_{\mathbb{R}^{+}} \exp \left(-\frac{x^{2}+15 t^{2}}{20}\right) \cos \left(\frac{x+2 \varepsilon t}{t}\right) \sin \left(\frac{x+2 \varepsilon t}{t}\right) \cdot \frac{k}{\sqrt{t}} \exp \left(-\frac{(x+2 \varepsilon t)^{2}}{80 t}\right) d \varepsilon
$$

Where $\varepsilon=\varepsilon_{4}$.

Now, as another example, we consider the KdV equation $u_{t}+u_{x x x}+u u_{x}=0$. First, the general solution from the Lie symmetry methods as follows:

$$
\tilde{u}(x, t ; \varepsilon)=\exp \left(-2 \varepsilon_{4}\right) u\left(\exp \left(-\varepsilon_{4}\right) x-\varepsilon_{1}, \exp \left(-\varepsilon_{4}\right) t-\varepsilon_{2}\right) .
$$

For the vector field $v_{1}=\partial_{x}+2 \partial_{t}$ with the differential invariant $(x-2 t)$ and for mother wavelet $\psi_{1}$, the wavelet type transform as follows:

$$
\iint \frac{4}{5} \exp \left(-\frac{\left(x-2 \varepsilon_{1}\right)^{2}+\left(t-\varepsilon_{2}\right)^{2}}{2}\right) \sin \left(\frac{\partial\left(x-2 t+2\left(\varepsilon_{2}-\varepsilon_{1}\right)\right)}{2}\right) \tilde{u}(x, t ; \varepsilon) d \varepsilon_{1} d \varepsilon_{2},
$$

The calculations are similar for $\psi_{2}$. By considering the invariant solution as follows:

$$
u(x, t)=6 \operatorname{sech}^{2}\left[\frac{\sqrt{2}}{2}(x-2 t)+\delta\right] .
$$

The wavelet types transform corresponding to $\psi_{2}$ as below:

$$
\iint \frac{24}{5} \exp \left(-\frac{\left(x-2 \varepsilon_{1}\right)^{2}+\left(t-\varepsilon_{2}\right)^{2}}{2}\right) \cos \left(\frac{\pi A}{2}\right) \cdot \operatorname{sech}^{2}\left[\frac{\sqrt{2}}{2} A+\delta\right] d \varepsilon_{1} d \varepsilon_{2},
$$

where all integrals are calculated on $\mathbb{R}^{+} \times \mathbb{R}^{+}$.

In the following, the generalized FKPP equations are analyzed by WTTM. Under the scaling symmetry group with parameter $\lambda=\exp (\varepsilon)$ and for invariants $\left(x / t, x / t^{2}\right)$, by the mother wavelet ${ }_{3}$ the general solution as follows:

$$
\tilde{u}(x, t ; \varepsilon)=\exp (2 \varepsilon) u(\exp (-\varepsilon) x, \exp (-2 \varepsilon) t),
$$

Thus,

$$
\psi_{3}(x, t ; \varepsilon)=\exp \left(-\frac{\exp (2 \varepsilon) x^{2}+15 \exp (4 \varepsilon) t^{2}}{20}\right) \cos \left(\frac{\pi x}{\exp (\varepsilon) t}\right) \sin \left(\frac{\pi x}{\exp (\varepsilon) t}\right),
$$
and we get,

$$
\int_{\mathbb{R}^{+}} \exp \left(2 \varepsilon-\frac{\exp (2 \varepsilon) x^{2}+15 \exp (4 \varepsilon) t^{2}}{20}\right) \cos (B) \sin (B) \cdot u(\exp (-\varepsilon) x, \exp (-2 \varepsilon) t) d \varepsilon,
$$

Where $B=\frac{\pi x}{\exp (\varepsilon) t}$. Now, we replace $u(x, t)$ by the solution of GFKPP equation, for example, in the primary version of GFKPP equation (that is $u_{t}=u_{x x}+u-u^{2}$ ), the analytic solution is:

$$
u(x, t)=\frac{1}{1+F(x) \exp (-t)} .
$$

So, we have:

$$
\int_{\mathbb{R}^{+}} \frac{\exp \left(2 \varepsilon-\frac{\exp (2 \varepsilon) x^{2}+15 \exp (4 \varepsilon) t^{2}}{20}\right) \cos (B) \sin (B)}{1+F(\exp (\varepsilon) x) \exp (-\exp (2 \varepsilon) t)} d \varepsilon,
$$

and for the generalized version with $f(x)$, we get,

$$
\int_{\mathbb{R}^{+}} \exp \left(2 \varepsilon-\frac{\exp (2 \varepsilon) t}{f(\exp (\varepsilon) x)}-\frac{\exp (2 \varepsilon) x^{2}+15 \exp (4 \varepsilon) t^{2}}{20}\right) \cos (B) \sin (B) \cdot G(\exp (\varepsilon) x) d \varepsilon,
$$

Hence, if the functions $f(x), F(x), G(x)$ are determined, we can easily obtain the wavelet invariant solutions.

\section{Conclusions and Future Works}

In this paper, we proposed the novel method based on the wavelets and symmetry groups. We constructed new mother wavelets with two variables according to the differential invariants (in the different style), then calculated the corresponding wavelet type transforms, applied them on the invariant solutions of PDEs and hereafter, the solutions were obtained. Unlike other applications of wavelets and wavelet transforms in the numerical analysis and other fields of the differential equations, our method results in the analytic and exact solutions. Indeed, we have relied upon constructing solutions from other solutions, on the other words, WTTM uses the existing solutions (from every method without any exception), then acts on them and produces the new solutions. In this method, the crucial step is to provide the proper mother wavelet according to the differential invariants, therefore this method can be used to solve the PDEs that equivalence methods (like the Lie symmetry method) can be applied on those. In fact, our contributions are new wavelets and the new wavelet method that with its wavelet type transform acts on the solution manifolds and produces new solutions from existing ones. By this method, we can produce a considerable range of integral operators. In the future works, by implementing WTTM on other PDEs, we will propose the suitable mother wavelets for every differential invariant and symmetry group and generalized the method for solving PDEs at every order and every number of independent variables.

\section{Acknowledgements}

We wish to thank professor Megeredich Toomanian for his valuable tips, advises and suggestions in the field of harmonic analysis. The authors appreciate him for benefiting from his precious experiences and knowledge.

\section{References}

1. Debnath L (2002) Wavelet Transforms and Their Applications, Springer.

2. Bluman GW, Kumei S (1989) Symmetries and Differential Equations, Springer.

3. Craddock M (2015) Fourier type transforms on Lie symmetry groups. Journal of Mathematical Physics, AIP 56(9): 10.1063-1.4929653.

4. Olver PJ (1993) Applications of Lie Groups to Differential Equations, Springer.

5. Polyanin AD, Zaitsev VF (2004) Handbook of Nonlinear Partial Differential Equations, Chapman \& Hall CRC.

6. Nadjafikhah M (2008) Lie Symmetries of Inviscid Burger's Equation, Advances in Applied Clifford Algebras, Springer 19(1): 101-112.

7. Misiti MI, Misiti Y, Oppenheim G, Poggi JM (2007) Wavelets and Their Applications, ISTE Ltd.

8. Yang X, Xu D, Zhang H, Nicolson C (2012) Quasi-wavelets method for solving fourth order partial integro differential equation with a weakly singular kernel. Journal of Computational Physics, Elsevier.

9. Ali ST, Antoine JP, Gazeau JP (2014) Coherent States, Wavelets and their Generalization, Springer. 